\title{
Calcul et mesure de la température d'un plasma à l'aide des raies d'émission du cuivre
}

\author{
M. Sassi, L. Pierre, J. Bénard et C. Cahen (*) \\ Direction des Etudes et Recherches, Electricité de France, Département Retour d'Expérience, \\ Mesures, Essais, 25 allée privée, 93206 Saint Denis Cedex 03, France
}

(Reçu le 24 mars 1993, révisé le 6 septembre 1993, accepté le 19 octobre 1993)

\begin{abstract}
Résumé. - L'utilisation des raies d'émission du cuivre pour la mesure de la température dans les plasmas est présentée. Cet article expose les problèmes posés par l'étude des températures dans les plasmas stationnaires et instationnaires. Dans le premier cas, on a été concerné par la mesure de la température dans le jet d'une torche à plasma industrielle ( $2 \mathrm{MW})$. Le plasma est stationnaire et la géométrie de la mesure permet l'inversion d'Abel pour remonter aux profils de température dans le jet. Les limitations de la méthode de mesure sur les bords du jet ainsi que le refroidissement du plasma par bouffées d'air froid sont discutés. Dans le second cas, un plasma instationnaire d'un four à arc $(1,4 \mathrm{MW})$ a été étudié. Cette étude nous a permis de mettre en relief le rôle de la dynamique du détecteur et la façon de l'adapter à la dynamique du milieu observé. Les mesures présentées permettent de conclure sur une température probable au voisinage immédiat de l'arc. Au terme de l'article, on conclut sur la validité du cuivre comme traceur pour la mesure de température dans les plasmas.
\end{abstract}

\begin{abstract}
The use of copper emission lines for temperature measurement in plasma environments is presented. The article features the problems encountered with such a measurement method when used in a stationnary and a non-stationnary plasma. In the first case, we were concerned with the temperature measurements in the jet of a $2 \mathrm{MW}$ industrial plasma torche. The plasma was stationnary and the measurement geometry allowed the use of the Abel inversion method to recover the temperature profiles in the plasma jet. The limitations of the measurement method on the jet boundaries as well as the cooling of the plasma by entrainement of cold air are discussed. In the second case, a non-stationnary plasma in a $1.4 \mathrm{MW}$ electric furnace was studied. This study allowed to feature the role of the detector dynamics as related to the dynamics of the observed medium. The obtained measurements show only a probable temperature in the immediate proximity of the arc. Finally, we conclude on the validity of copper as a tracer and the use of spontaneous emission spectroscopy, an easy method to implement, compared to other sophisticated temperature measurement methods.
\end{abstract}

(*) Auteur pour la correspondance. 


\section{Notation.}

$A . B, \sigma$ paramètres d'une gaussienne

$E_{1} \quad$ énergie du niveau i

$f_{\mathrm{y}} \quad$ force d'oscillateur de la raie $\mathbf{i} \rightarrow \mathbf{j}$

$g_{1} \quad$ dégénérescence du niveau $\mathrm{i}$

$I_{1} \quad$ intensité de la raie $\mathrm{i} \rightarrow \mathrm{j}$

$I(y) \quad$ intensité sur une corde

$I^{\prime}(y)$ dérivée de $I(y)$

$J(r) \quad$ intensité radiale

$k$ constante de Boltzmann

$r \quad$ rayon

$R \quad$ rayon du jet de plasma

$T \quad$ température

$x, y, y_{1}$ coordonnées rectangulaires

$\lambda_{1 \mathrm{j}} \quad$ longueur d'onde de la raie $\mathrm{i} \rightarrow \mathrm{j}$

\section{Introduction.}

Lorsqu'il effectue des mesures de températures sur un plasma d'arc électrique, l'expérimentateur se trouve confronté à une large gamme de températures : typiquement de $2500 \mathrm{~K}$ à $20000 \mathrm{~K}$. Les valeurs les plus élevées se rencontrent dans l'arc (effet Joule), les plus basses se trouvent loin de celui-ci dans le milieu réchauffé par les différents phénomènes de transport de la chaleur. Bien que d'utilisation très classique, la spectroscopie d'émission se révèle être une bonne méthode d'investigation des champs de température dans ces plasmas, en particulier grâce aux développements technologiques des détecteurs. Deux cas de figures principaux se présentent. Dans le premier cas, on étudie les températures loin de l'arc, où la stabilité du phénomène autorise des mesures locales. Le second est la recherche de températures sur l'arc, où le phénomène est localisé mais instable et seules des mesures globales sont possibles. Chacune des deux situations présente des difficultés propres: problèmes de restitution numérique pour la première, problèmes de dynamique pour la seconde.

Le premier défi de la mesure de température par spectroscopie d'émission est le choix des raies thermométriques. Le plasma est composé d'ions, d'électrons, d'atomes et de molécules. Les gaz atomiques émettent des spectres de raies. Les gaz moléculaires émettent des spectres de bandes généralement pas résolues. De plus, les bandes ne sont pas toujours isolées et leur chevauchement pose un problème d'identification et de mesure. Dans les plasmas, la température est généralement très élevée, les molécules sont dissociées et la mesure est souvent effectuée sur les raies atomiques. Les émissions atomiques peuvent provenir du gaz plasmagène ou d'un élément traceur. La plupart des générateurs de plasma utilisent des électrodes de cuivre en raison de sa bonne tenue aux hautes températures. La température atteinte aux points d'accrochage vaporise un peu de métal qui se retrouve alors dans le milieu environnant.

Les méthodes spectroscopiques de mesure permettant de déterminer la température (intensité absolue des raies spectrales, intensités relatives des raies, intensité du fond continu, profil des raies, etc.) ont été largement utilisées et discutées dans la littérature [1-4].

Une recherche systématique sur les caractéristiques spectrales dans la fenêtre d'observation de l'ensemble spectroscope-détecteur $(15 \mathrm{~nm})$ a conduit à la sélection de quatre raies 
d'émission du cuivre. Aucun spectre bien résolu d'espèce moléculaire n'a été observé. Ces raies du cuivre ne se désexcitent pas vers le niveau fondamental et ne présentent donc pas d'auto-absorption. Pour une mesure instantanée de la température, ces raies sont particulièrement bien adaptées puisque l'on peut les enregistrer simultanément. D'autres auteurs les ont déjà utilisées pour des mesures de température dans les plasmas $[5,6]$.

Sous l'hypothèse de l'équilibre thermodynamique local (ETL), l'intensité d'une raie d'émission est reliée à la température par la relation :

$$
I_{\mathrm{l}]}=c f_{\mathrm{lj}} g_{1} \frac{1}{\lambda_{1 \mathrm{j}}^{4}} \exp \left(-\frac{E_{1}}{k T}\right)
$$

où $c$ est une valeur numérique reprenant les constantes, les corrections caractérisant la chaîne de mesure et son efficacité. La température se déduit de la pente de la droite de Boltzmann, et la précision sur cette déduction dépend du nombre de points pour construire cette droite et de leur grande distribution sur les différents niveaux d'énergie de l'espèce étudiée. Le meilleur compromis entre les intensités d'émission et les déformations des raies par auto-absorption reste l'utilisation de raies du cuivre dans la partie visible du spectre même si la distribution d'énergie pour ces quatre raies est très limitée (trois niveaux d'énergie concernés dont deux très proches). La droite de Boltzmann ne s'appuie pratiquement que sur trois points dont deux très proches et donc la preuve de l'ETL est discutable. Il faut nécessairement confirmer cette hypothèse par des mesures indépendantes. Toutefois l'utilisation de ces trois points permet d'augmenter la précision sur la restitution de la pente et donc de la température.

La figure 1 montre la simulation des intensités relatives des raies observées en fonction de la température et fait apparaître trois zones différentes qui confirment l'utilisation possible de la méthode de mesure sur une large gamme de températures : 2 500-20 000 K. On voit en effet que sur cette plage de température, le rapport des intensités des raies extrêmes évolue d'une manière significative (Fig. 2).

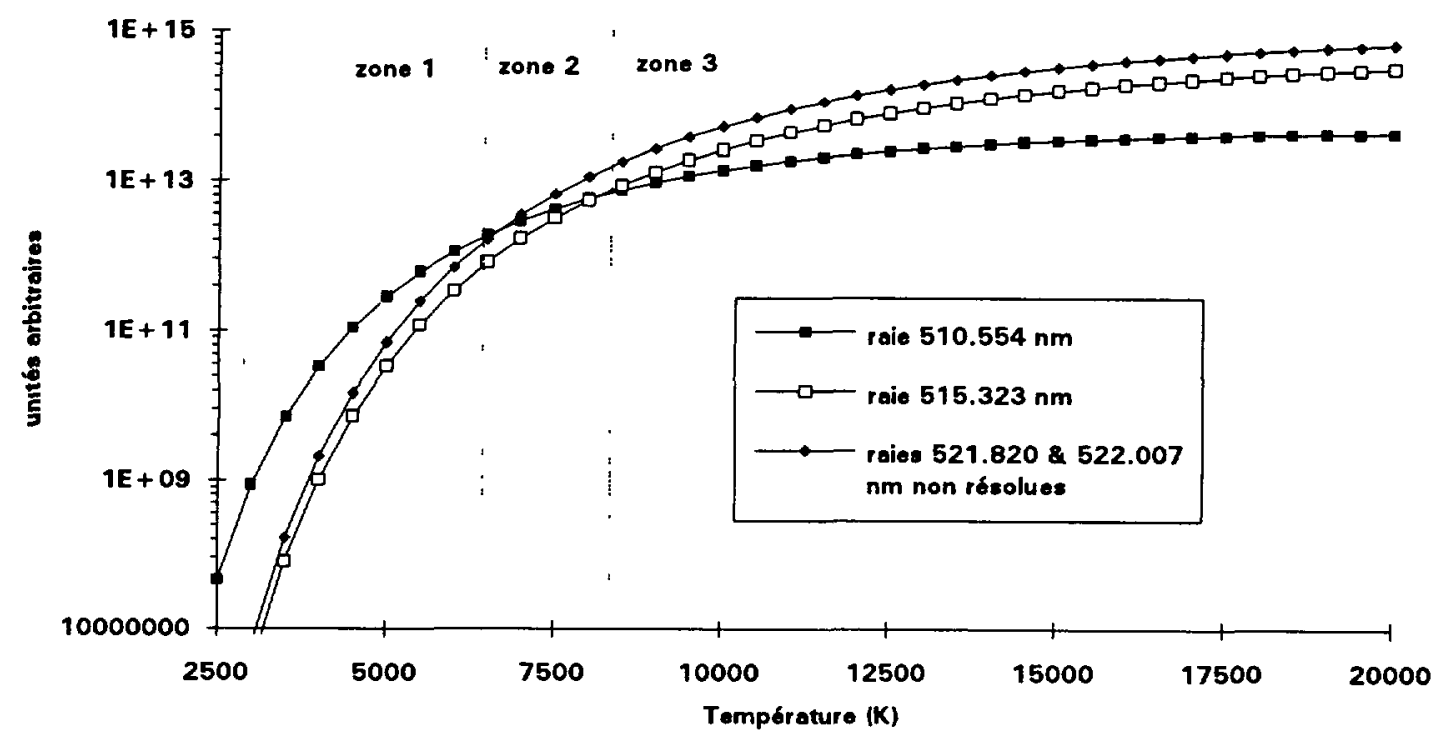

Fig. 1. - Intensité des raies d'émission du cuivre, évolution avec la température.

[Copper line intensities as a function of temperature.] 


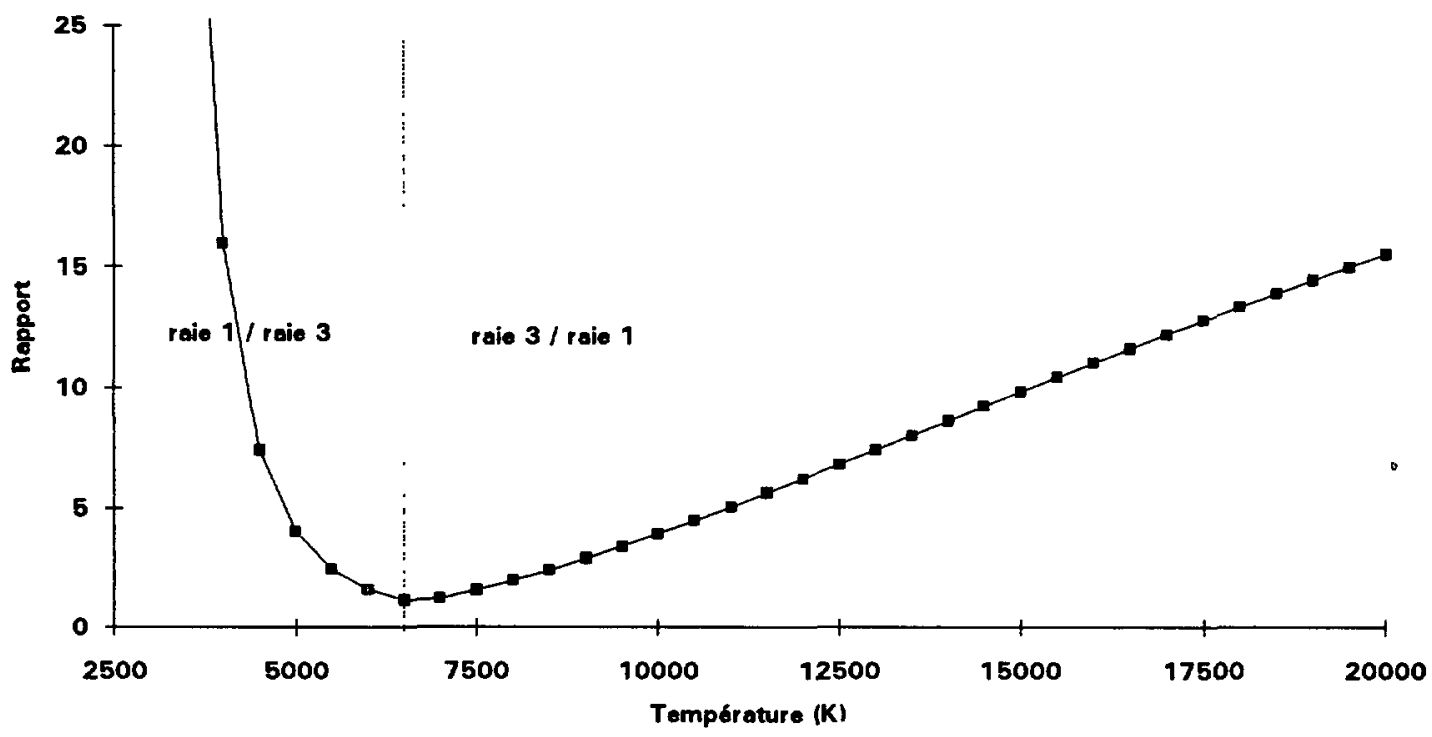

Fig. 2. - Evolution avec la température du rapport des raies 1 et 3.

[Intensity ratio of lines 1 and 3 as a function of temperature.]

L'incertitude principale de la mesure est reliée aux valeurs numériques disponibles des forces d'oscillateur du cuivre données dans la littérature avec une incertitude de $25 \%$ [7, 8]. Un balayage des différentes valeurs possibles (Tab. I) a révélé d'importantes variations des températures absolues (rien ne change quant aux mesures relatives). Les produits des dégénérescences par les forces d'oscillateurs, comprises dans les limites de l'incertitude, permettent d'obtenir des températures minimales, moyennes et maximales à partir d'une même série de mesure. Cette même incertitude se retrouve sur la mesure à moins de connaître localement une valeur absolue de la température. Cette valeur, déterminée par une autre méthode, servira de référence pour une mise à l'échelle de l'ensemble des températures.

Tableau I. - Les propriétés physiques des transitions intéressantes.

[The physical properties of the selected copper transitions.]

\begin{tabular}{|c|c|c|c|}
\hline Transition & Longueur d'onde $(\mathrm{nm})$ & $\begin{array}{c}\text { Force d'oscillateur } \\
\text { de la transition } \\
\text { d'après [4] }\end{array}$ & $\begin{array}{c}\text { Dégénérescence } \\
\text { du niveau de départ }\end{array}$ \\
\hline${ }^{2} \mathrm{D}_{3 / 2} \rightarrow{ }^{2} \mathrm{P}_{1 / 2}^{0}$ & 515,323 & $0,6 \pm 25 \%$ & 4 \\
\hline${ }^{2} \mathrm{P}_{3 / 2}^{0} \rightarrow{ }^{2} \mathrm{D}_{3 / 2}^{0}$ & 510,554 & $0,004 \pm 25 \%$ & 4 \\
\hline${ }^{2} \mathrm{D}_{3 / 2} \rightarrow{ }^{2} \mathrm{P}_{3 / 2}^{0}$ & 522,007 & $0,07 \pm 25 \%$ & 4 \\
\hline${ }^{2} \mathrm{D}_{5 / 2} \rightarrow{ }^{2} \mathrm{P}_{3 / 2}^{0}$ & 521,820 & $0,6 \pm 25 \%$ & 6 \\
\hline
\end{tabular}




\section{Mesure de la température dans un jet de plasma stationnaire.}

2.1 LE CONTEXTE. - Cette étude concerne la restitution de profils de température en sortie immédiate de la torche pour fournir des données d'entrées réalistes au modèle d'aérothermodynamique qui décrit la dilution de jet de plasma dans l'air. Ces études de dilution ont été conduites sur le même générateur de plasma. La validation de la prédiction des modèles a été obtenue par des mesures de température par Diffusion Raman anti-Stokes Cohérente (DRASC) et de vitesse par vélocimétrie laser $[9,10]$. La mesure de température $\mathbf{n}$ était pas possible en sortie immédiate de la torche : la température peut atteindre $6000 \mathrm{~K}$ dépassant largement la limite de détectivité de la DRASC $(3000 \mathrm{~K})$. Il a fallu compléter ces mesures par une autre méthode de mesure qui, dans ce domaine de température, est principalement la spectroscopie d'émission plus facile de mise en œuvre.

2.2 LA TORCHE À PLASMA. - La figure 3 montre la torche à plasma qui se compose d'une électrode amont, d'une électrode aval, toutes les deux en cuivre, d'une chambre d'injection du gaz plasmagène, d'une bobine assurant la rotation du pied d'arc pour prévenir l'érosion des électrodes, d'une électrode d'amorçage et d'un circuit de refroidissement. L'injection de gaz plasmagène est tangentielle afin de créer un vortex pour assurer un transfert maximal d'énergie entre l'arc et le gaz. La torche de $2 \mathrm{MW}$ de puissance est alimentée en courant continu par quatre modules de redresseur délivrant chacun au maximum $1000 \mathrm{~A}-1000 \mathrm{~V}$. La pression interne de la torche varie de 1 à 5 bars et le débit nominal d'air plasmagène est de $600 \mathrm{Nm}^{3}$ /heure. Le refroidissement de la torche se fait par eau déminéralisée à un débit de $50 \mathrm{~m}^{3} /$ heure. La trace d'érosion du pied de l'arc sur l'électrode aval est approximativement située à $35 \mathrm{~cm}$ de la sortie de la torche de $5 \mathrm{~cm}$ de diamètre intérieur. Cela permet de supposer un bon mélange des vapeurs de cuivre provenant de l'électrode et du gaz plasmagène en sortie de torche.

2.3 LA CHAÎNE DE MESURE. - La figure 4 présente la chaîne de mesure de spectroscopie d'émission. C'est un système afocal se composant de deux lentilles de $1000 \mathrm{~mm}$ de focale

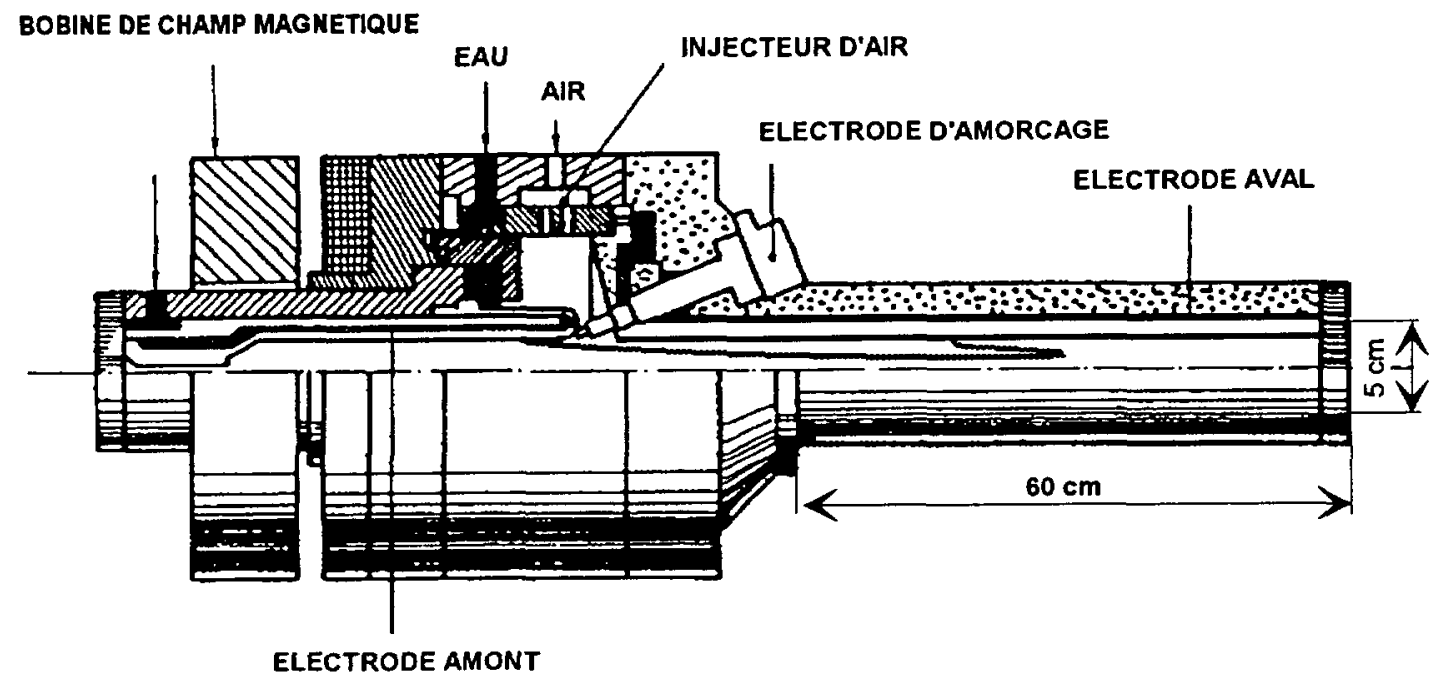

Fig. 3. - La torche à plasma $2 \mathrm{MW}$.

[The $2 \mathrm{MW}$ plasma torch.] 


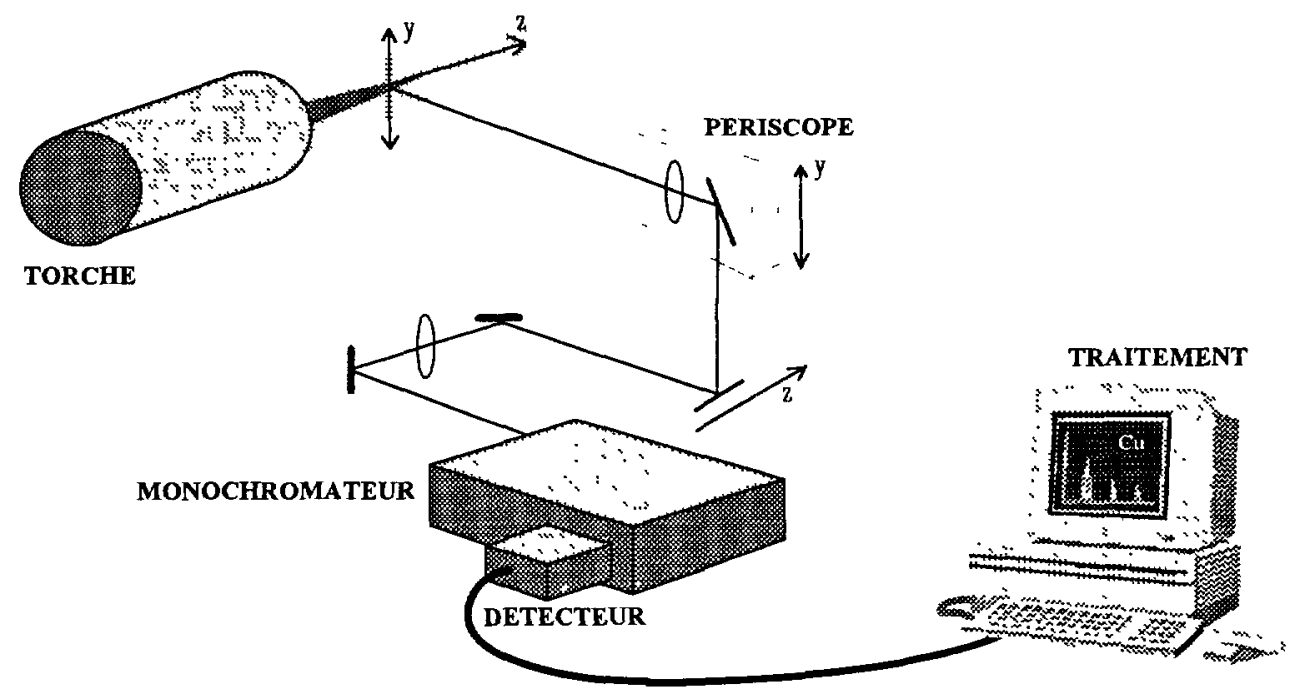

Fig. 4. - Montage expérimental autour de la torche à plasma.

[Experimental set-up around the plasma torch.]

(grandissement $\times 1$ ) et de miroirs de renvoi. Le volume de mesure représenté par l'image de la fente d'entrée du spectroscope fait $1 \mathrm{~cm}$ de long et $25 \mu \mathrm{m}$ de haut. Le signal est dispersé par le spectroscope de $640 \mathrm{~mm}$ de focale équipé d'un réseau de 1200 traits/mm et est détecté par une caméra intensifiée de $578 \times 384$ pixels. Les déplacements suivant les axes y et $z$ sont assurés par des tables micrométriques sans déréglage de l'alignement. Le temps d'exposition de la caméra est de $100 \mathrm{~ms}$ et le calculateur de contrôle assure l"intégration sur la hauteur de la fente. Chaque mesure correspond à une moyenne de 100 spectres consécutifs. Les intensités affectées aux raies d'émission et le bruit de fond correspondent à une intégration sur une fenêtre de 40 pixels de large.

2.4 LA GÉOMÉTRIE DE MESURE ET L'INVERSION D'ABEL. - Le jet du plasma présente une symétrie cylindrique et la géométrie de mesure est montrée sur la figure 5 . On obtient une mesure intégrée suivant une corde d'intensité $I(y)$ non représentative de l'intensité locale

$$
I(y)=2 \int_{y}^{R} I(r) \frac{r \mathrm{~d} r}{\left(r^{2}-y^{2}\right)^{1 / 2}} .
$$

Pour accéder à la température du plasma, il faut remonter à l'intensité radiale des raies du cuivre puis utiliser la méthode du graphique atomique de Boltzmann. Le traitement mathématique est donc l'inversion d'Abel

$$
J(r)=-\frac{1}{\pi} \int_{r}^{R} I^{\prime}(y) \frac{y \mathrm{~d} y}{\left(y^{2}-r^{2}\right)^{1 / 2}} .
$$

Nous avons comparé les différentes méthodes citées dans la littérature [11-24] et choisi et adapté la méthode proposée par Deutsch [18]. Cette méthode a été recommandée pour les 


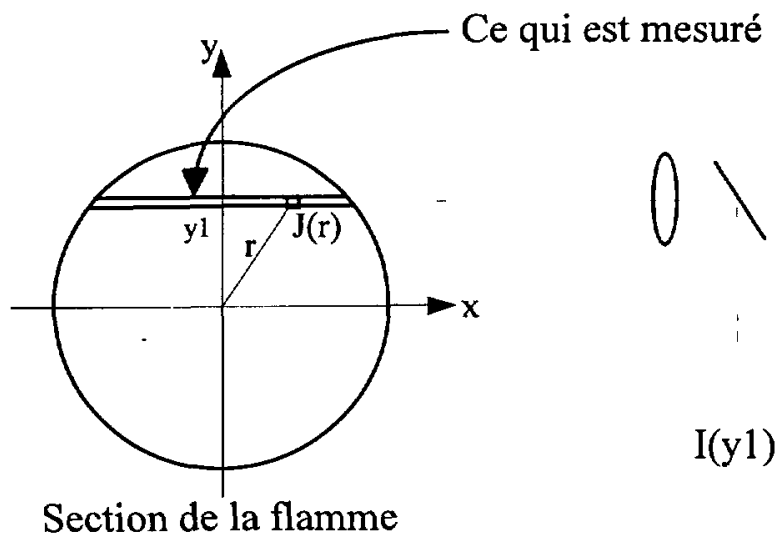

Fig. 5. - Géométrie de la mesure.

[Geometry of the measurement.]

intensités $I(y)$ en forme de cloche et validée sur un plasma inductivement couplé. C'est une approximation de $I(y)$ par la fonction

$$
I_{N}(y)=P_{N}\left(y^{2}\right) \exp \left(-\alpha y^{2}\right)=\left(\sum_{n=0}^{N} a_{n} y^{2 n}\right) \exp \left(-\alpha y^{2}\right)
$$

où $P_{N}(x)$ dénote un polynôme de degré $N$ en $x$. L'inverse d'Abel se calcule d'une façon analytique évitant les problèmes d'évaluation de la dérivée et des singularités. L'approximation non linéaire de $I(y)$ a été faite par la méthode de Levenberg-Marquardt d'après l'algorithme décrit dans Press et al. [25]. On a reconstitué les tests et les résultats de l'auteur de la méthode et trouvé de très bons accords.

2.5 TRAitement des données et RÉSultats. - On a utilisé la méthode d'inversion d'Abel décrite ci-dessus avec $N=2$ et $N=5$. Vu le faible nombre de points de mesure $(\sim 15)$, on a remarqué que les profils restent proches des gaussiennes et les coefficients du polynôme qui raffine l'approximation gaussienne, restent très petits dans les deux cas. On a

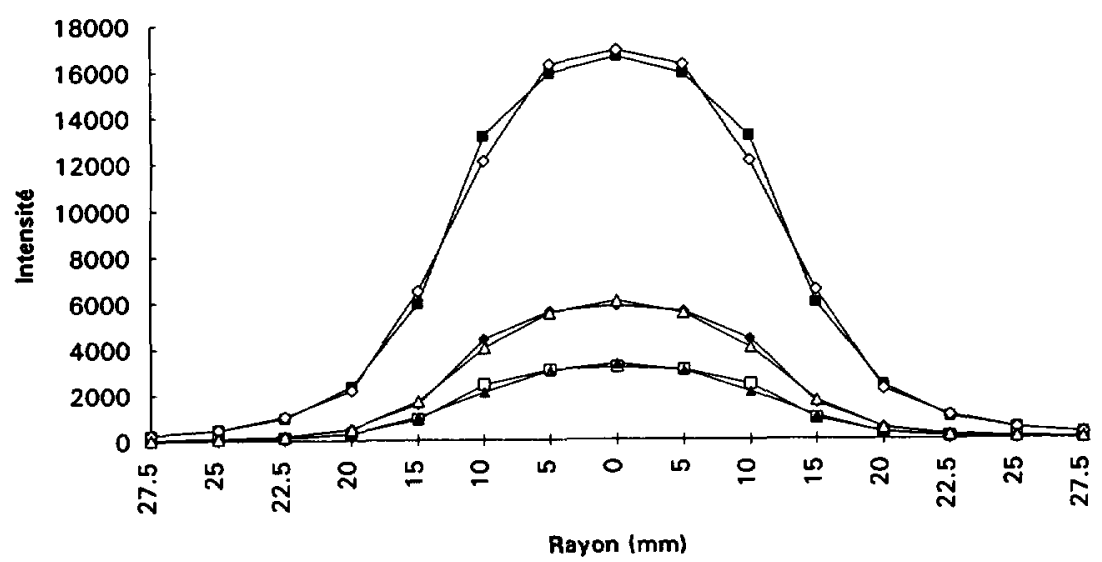

Fig. 6. - Approximation des courbes expérimentales par des gaussiennes multipliées par des polynômes $(N=2)$.

[Approximation of the experimental curves by the products of Gaussians and polynomes $(N=2)$.] 


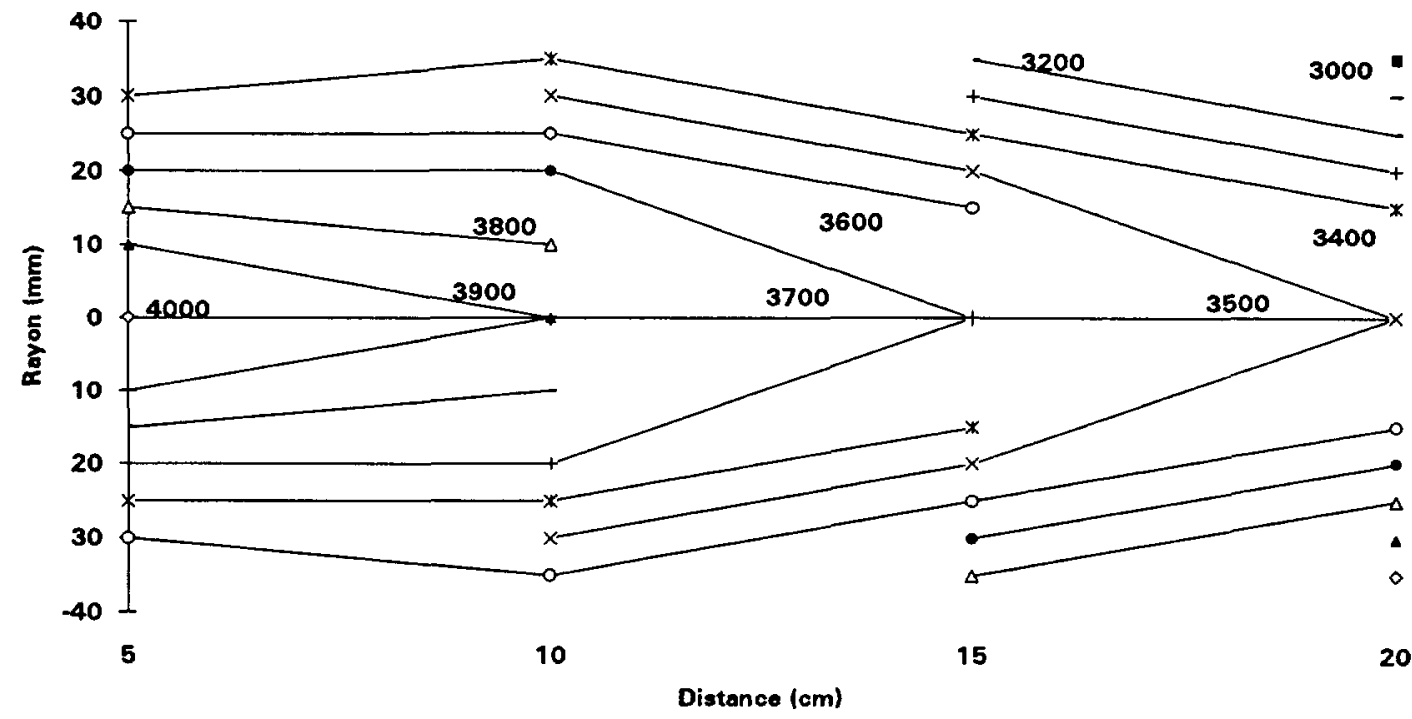

Fig. 7. - Isothermes du jet, Intensité $700 \mathrm{~A}$, Débit $108 \mathrm{~g} / \mathrm{s}$.

[Isotherms in the plasma jet, Intensity $=700 \mathrm{~A}$, Mass flow rate $=108 \mathrm{~g} / \mathrm{s}$.]

donc traité les données expérimentales avec $N=2$. Un exemple des profils des intensités mesurées des trois raies de cuivre et leurs approximations est donné sur la figure 6 . Après inversion, on obtient un profil radial de la température, et la figure 7 expose les isothermes dans le jet du plasma.

L'hypothèse de l'ETL a été vérifiée de deux manières indirectes. Un bilan de transfert de chaleur sur la torche, basé sur des mesures cies températures de l'eau de refroidissement, donne une température de gaz plasmagène à la sortie de la torche proche de $4000 \mathrm{~K}$. Des mesures locales intrusives par sonde enthalpique ont été effectuées à la sortie de la torche et ont montré des températures de l'ordre de $4200 \mathrm{~K}$. La sonde a été utilisée pour fournir des mesures pour comparaison avec les mesures spectroscopiques. Malheureusement elle n'a pas tenu dans ce milieu hostile et cette partie de l'étude a été différée. Néanmoins ces deux vérifications ont conforté l'hypothèse de l'ETL.

La forme analytique de la fonction d'approximation suppose une décroissance à zéro du profil d'intensité sur les bords, et selon les cas de mesure, on a pu observer des températures qui remontaient sur la périphérie. Ceci est dû à la décroissance incorrecte de l'un des profils d'approximation sur les bords. Ces points ont été rejetés.

La comparaison entre les isothermes mesurés et ceux du modèle aérothermodynamique a montré un très bon accord sur l'axe du jet, tandis que sur les bords, les températures « spectroscopiques » sont plus élevées que celles prédites par le calcul. Ce désaccord peut être attribué à deux phénomènes largement discutés et validés par Pfender et ses collaborateurs [2634]. Le premier concerne les déviations entre la mesure et le modèle à la sortie de la torche où le jet du plasma est supposé être laminaire. A cause de forts gradients radiaux, les électrons très énergétiques diffusent vers les bords du jet induisant par collisions avec les atomes neutres des fortes populations dans des états excités. Les températures spectroscopiques peuvent être ainsi surestimées. Autrement dit, des déviations de l'ETL peuvent être présentes dans cette région. Malgré le fait que cette explication paraisse raisonnable, les températures mesurées de l'ordre de $4000 \mathrm{~K}$ n'entraînent pas des fortes densités électroniques et la mesure de celles-ci serait 


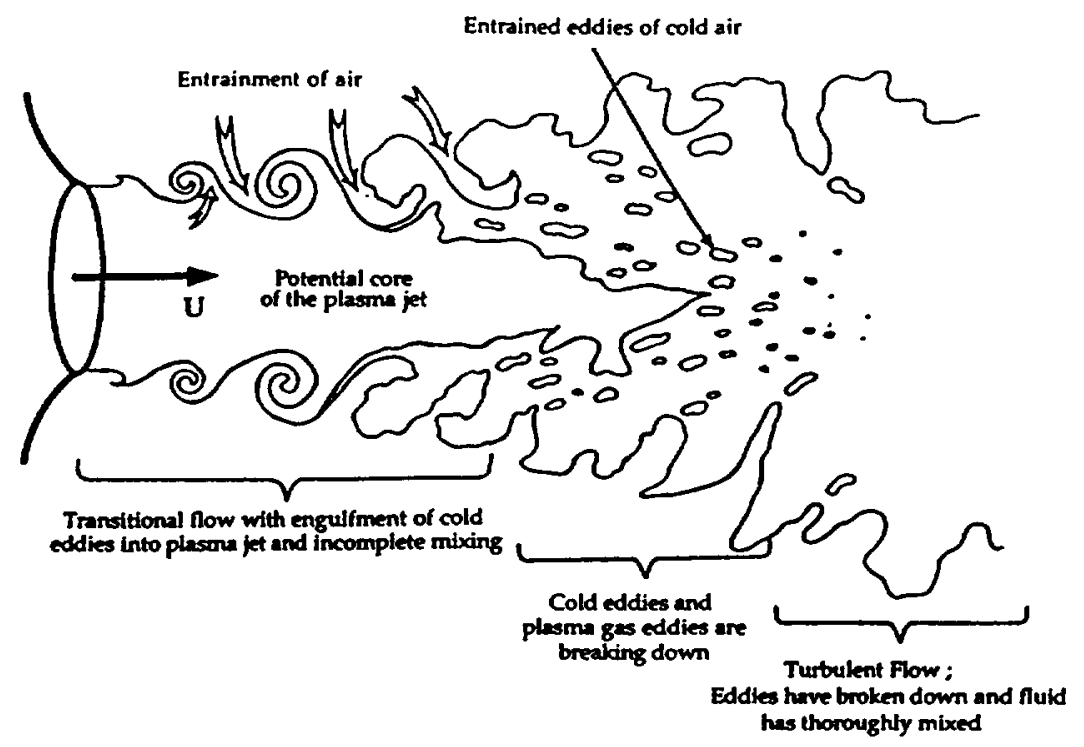

Fig. 8. - Refroidissement d'un jet de plasma par bouffées d'air froid, d'après Spores and Pfender.

[Cooling of a free plasma jet by entrainment of cold air, from Spores and Pfender.]

nécessaire pour valider ce point. Le second suppose que des bouffées d'air froid sont entraînées par le jet du gaz plasmagène chaud et persistent comme des poches d'air froid jusqu'à leur mélange homogène avec l'air chaud plus loin dans le jet (Fig. 8). La température spectroscopique du cuivre provient des seules zones d'air chaud et est plus élevée que celle prédite par le modèle qui suppose le bon mélange de deux gaz. Pour valider davantage cette hypothèse, on a fait les estimations suivantes:

- un cas test d'intensité $I(y)$ a été choisi et a fourni un profil de température $T$;

- à partir du diagramme d'énergie du cuivre, on calcule, pour chaque température et chaque raie, la fonction de partition du cuivre ;

- on calcule ensuite la concentration relative de cuivre, la concentration absolue étant elle supposée constante ;

- on calcule $J(r)$ (Eq. (3)), et par le calcul présenté précédemment (Eq. (2)), on remonte à $I\left(y^{\prime}\right)$;

- on compare le $I(y)$ calculé avec le $I(y)$ mesuré.

Si les $I(y)$ sont identiques, alors l'hypothèse est bonne, la concentration de cuivre est constante. Si les $I(y)$ sont différents, bien que $I(y)$ calculé soit issu des températures, ellesmêmes obtenues d'après $I(y)$ mesuré, c'est que la concentration de cuivre n'est pas constante.

La contradiction de l'hypothèse de la concentration constante apparaît très clairement sur la figure 9. On voit en effet que l'intensité calculée présente un profil parabolique, très éloigné du profil gaussien de l'intensité mesurée. On remarque la rapide croissance de l'écart entre les deux intensités. Celui-ci est maximum entre 15 et $25 \mathrm{~mm}$, ce qui indique une forte variation de la concentration sur la périphérie du jet. L'apparent accord des intensités pour les valeurs extrêmes, autour de 30-35 mm, vient de ce que, dans le calcul, on force l'intensité à s'annuler pour respecter la réalité physique observée. Cela ne signifie donc pas que la concentration 


\section{Comparaison $\mathrm{z}=5 \mathrm{~cm}$}

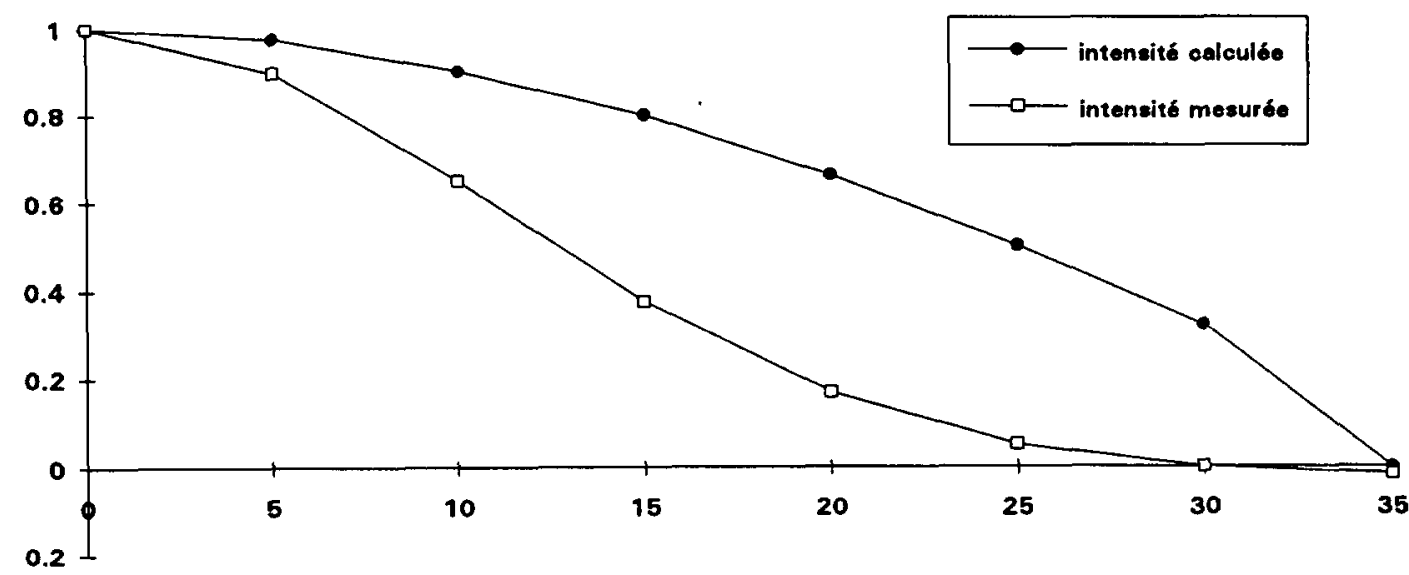

distance ou centre ( $\mathrm{mm}$ )

Fig. 9. - Comparaison des intensités calculée et mesurée, à $5 \mathrm{~cm}$ en sortie de torche.

[Comparison of the calculated intensity with the measured one, $5 \mathrm{~cm}$ after the torch exit.]

redevienne constante à $35 \mathrm{~mm}$ ! En revanche, la figure 10 montre que les deux intensités tendent à se rapprocher l'une de l'autre lorsqu'on s'éloigne de la sortie de la torche. Les deux gaz se mélangent donc davantage. Du fait de ce mélange, la concentration de cuivre tend à être uniforme sur le rayon du jet, tandis que son état d'excitation redevient significatif de la température moyenne du mélange. L'erreur sur la mesure diminue, ce que l'on constate sur la figure 10 .

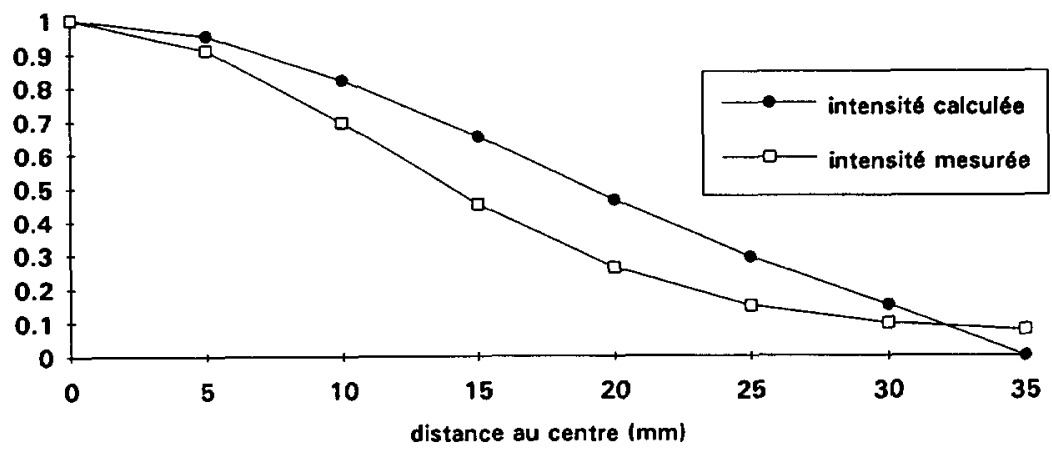

Fig. 10. - Comparaison à $20 \mathrm{~cm}$ en sortie de torche.

[Comparison of the calculated intensity with the measured one, $20 \mathrm{~cm}$ after the torch exit.] 


\section{Mesures de températures dans un arc électrique instationnaire.}

3.1 ConTEXTE. - Un exemple représentatif des problèmes de dynamique est offert par l'étude d'un four à arc électrique de puissance 1,4 MW à l'Institut de Recherches Sidérurgiques. Celui-ci, utilisé pour la fonte de ferrailles, est composé d'une enceinte calorifugée de forme sphérique d'environ $2 \mathrm{~m}$ de diamètre, d'une électrode en graphite de $30 \mathrm{~cm}$ de diamètre et d'une billette de quelques tonnes d'acier sur laquelle peut être déposé un traceur. Nous avons cherché à estimer numériquement les températures atteintes sur l'arc du four, afin d'effectuer un bilan énergétique et d'étudier les possibilités d'en augmenter le rendement. Des premières estimations faites par un code de calcul, basé sur deux températures, prédisent des températures d'électrons peu réalistes dans l'arc de $70000 \mathrm{~K}$ dans une atmosphère d'azote pur. Cependant, quelques pour-cents de $\mathrm{CO}_{2}$ (moins de $10 \%$ ) dans le plasma du four en présence de vapeurs métalliques abaisseraient cette température vers une température plus probable de $20000 \mathrm{~K}$. Cette hypothèse de présence de $\mathrm{CO}_{2}$ est réaliste puisque celui-ci peut être produit par les ferrailles contenant du carbone ou par l'érosion de l'électrode en graphite. Les sidérurgistes privilégient l'hypothèse du $\mathrm{CO}_{2}$ produit par les ferrailles. Des mesures préliminaires ont été entreprises, afin de conclure sur la température de l'arc et donc l'absence ou la présence de $\mathrm{CO}_{2}$.

L'observation de l'arc a été faite à travers une ouverture du four de $40 \mathrm{~cm} \times 40 \mathrm{~cm}$ (Fig. 11 ). Le système d'analyse de spectroscopie d'émission a été placé dans une chambre vitrée derrière des rideaux noirs pour protéger le matériel et le personnel du niveau de bruit sonore très élevé, de la forte intensité d'émission ultraviolette, et des particules de métal fondu éjectées du four. L'ouverture du four centrée sur l'arc est visée à une distance de $15 \mathrm{~m}$ par un téléobjectif de $300 \mathrm{~mm}$ placé devant le spectroscope. La fente d'entrée du monochromateur coupe une partie de $1 \mathrm{~cm}$ de haut et $25 \mu \mathrm{m}$ de large de l'image formée par l'objectif. Une densité de 4 est utilisée pour réduire l'intensité du signal et protéger la caméra $\mathrm{CCD}$. Le reste du système est celui utilisé sur la torche à plasma. L'ensemble (caméra, fente et téléobjectif) définit un volume de collection du signal au tour de l'arc de $20 \mathrm{~cm}$ de haut et $5 \mathrm{~cm}$ de large. Ainsi dix zones verticales d'observation de $2 \mathrm{~cm}$ ont été définies sur la caméra $2 \mathrm{D}$ afin d'avoir une information de la concentration des espèces émises sur l'axe de l'arc.

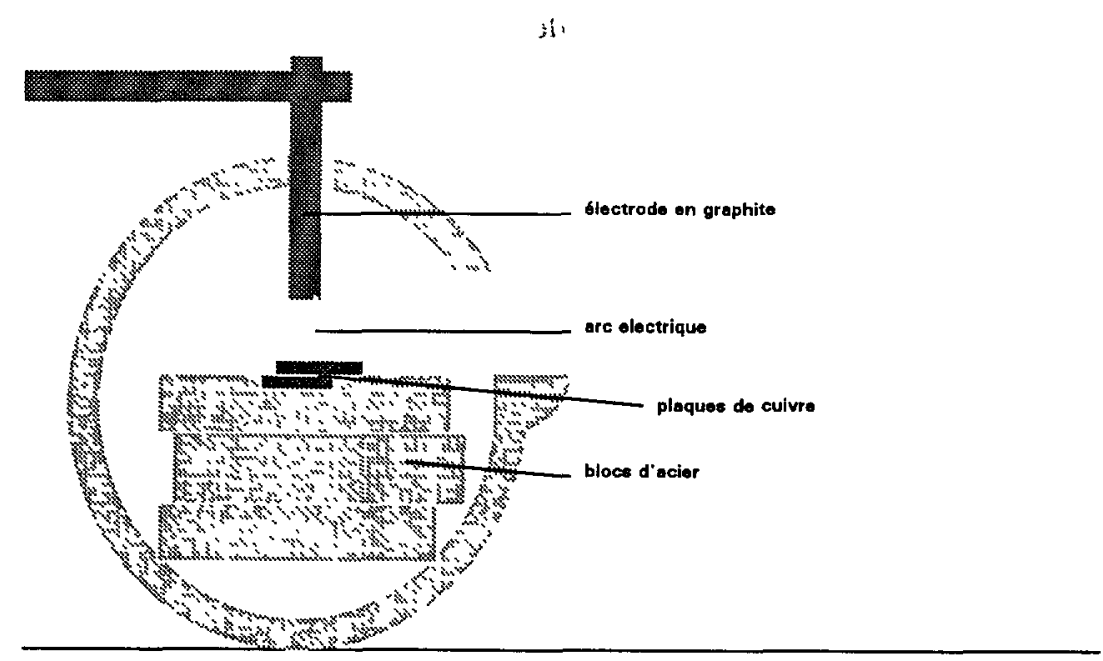

Fig. 11 - Configuration du four avec dépôt de cuivre.

[The oven with copper plates.] 


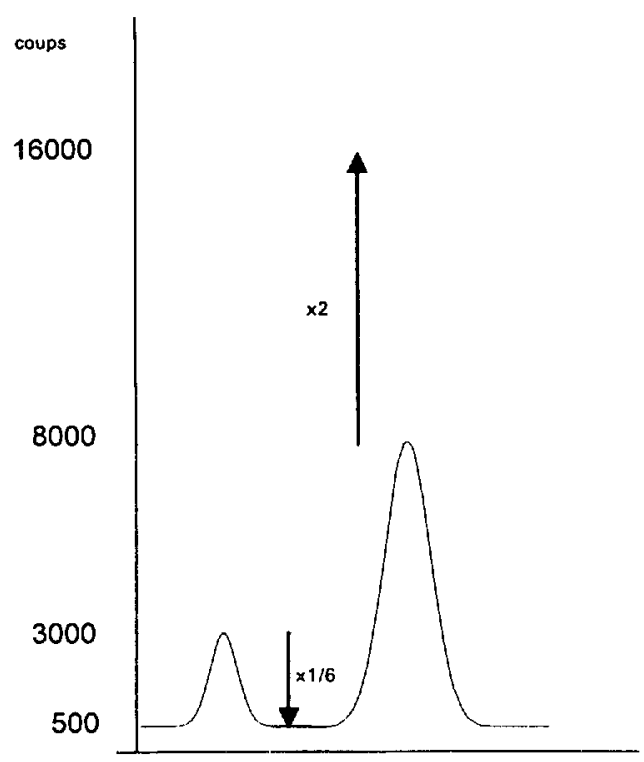

Fig. 12. - Influence de la dynamique sur la plage de températures mesurables.

[Influence of the camera dynamics on the range of measurable temperatures.]

Les détecteurs CCD, à $1 \mathrm{D}$ ou 2D, présentent souvent une dynamique de l'ordre de 14 bits. Celle-ci détermine les valeurs extrémales observables autour d'une température moyenne donnée. Il est important d'en tenir compte afin de ne pas fausser les mesures avec des raies saturées ou en dessous du niveau de bruit. Ceci est particulièrement vrai lorsque les phénomènes sont rapides et qu'il n'est alors pas possible d'adapter le gain du détecteur au signal. Une alternative possible serait un montage comprenant une division du signal (lame séparatrice) vers deux systèmes de détection, l'un à gain élevé et l'autre à gain faible. Avec un seul détecteur, une étude préliminaire permet de déterminer la configuration à adopter. Une

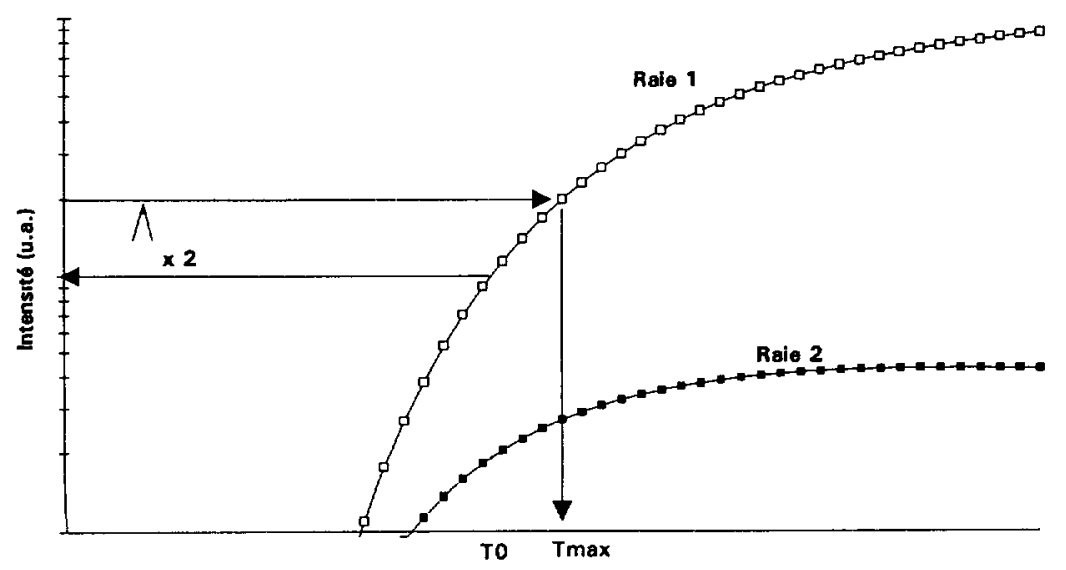

Fig. 13. - Détermination de la température maximale avant saturation.

[Determination of the maximum measurable temperature before detector saturation.] 


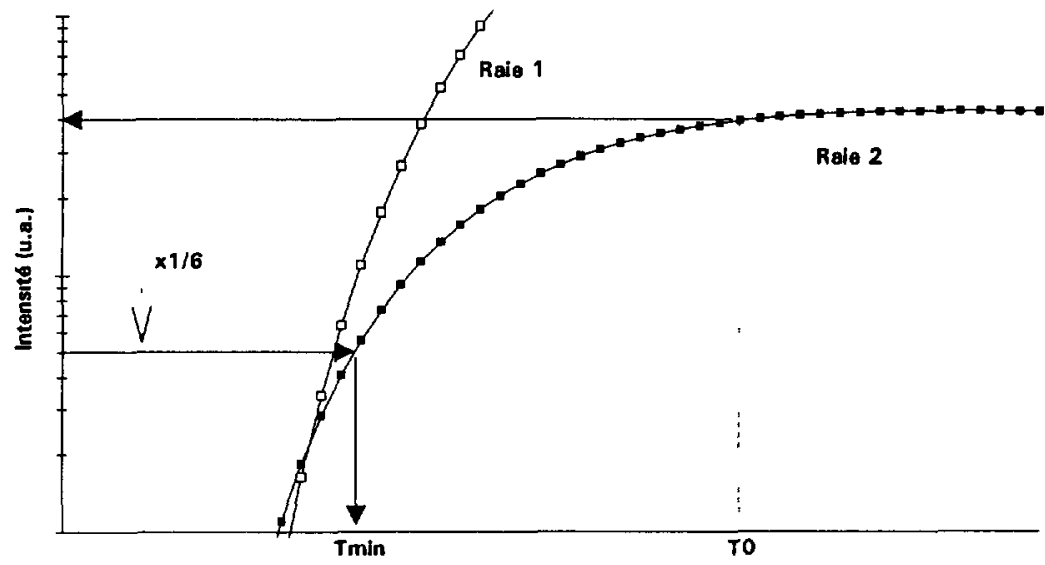

Fig. 14. - Détermination de la température minimale au-dessus du bruit.

[Determination of the minimum measurable temperature before reaching the detector noise limit.]

dynamique de 14 bits correspond à un nombre de coups maximum de 16384 . On pourra alors ajuster le gain de sorte que la raie la plus forte, à la température moyenne attendue $T_{0}$, corresponde au milieu de l'échelle, par exemple à 8000 coups, et prenant le niveau de bruit à 500 coups (Fig. 12). La température maximale mesurable avant saturation sera la température $T_{\max }$ qui double le nombre de coups enregistrés pour cette raie (Fig. 13); la température minimale $T_{\min }$ sera celle qui divise l'intensité de la raie la plus faible, au point de la faire passer sous le seuil de détection (Fig. 14).

3.2 RÉSULTATS. - Une expérience similaire conduite au Canada [35] a guidé le choix du titane comme traceur. La température de l'arc a été déterminée par le rapport d'une raie atomique et celle de son ion. Dans notre cas, l'usure rapide de la billette d'acier, d'environ

Raies du Fer ot du Titene

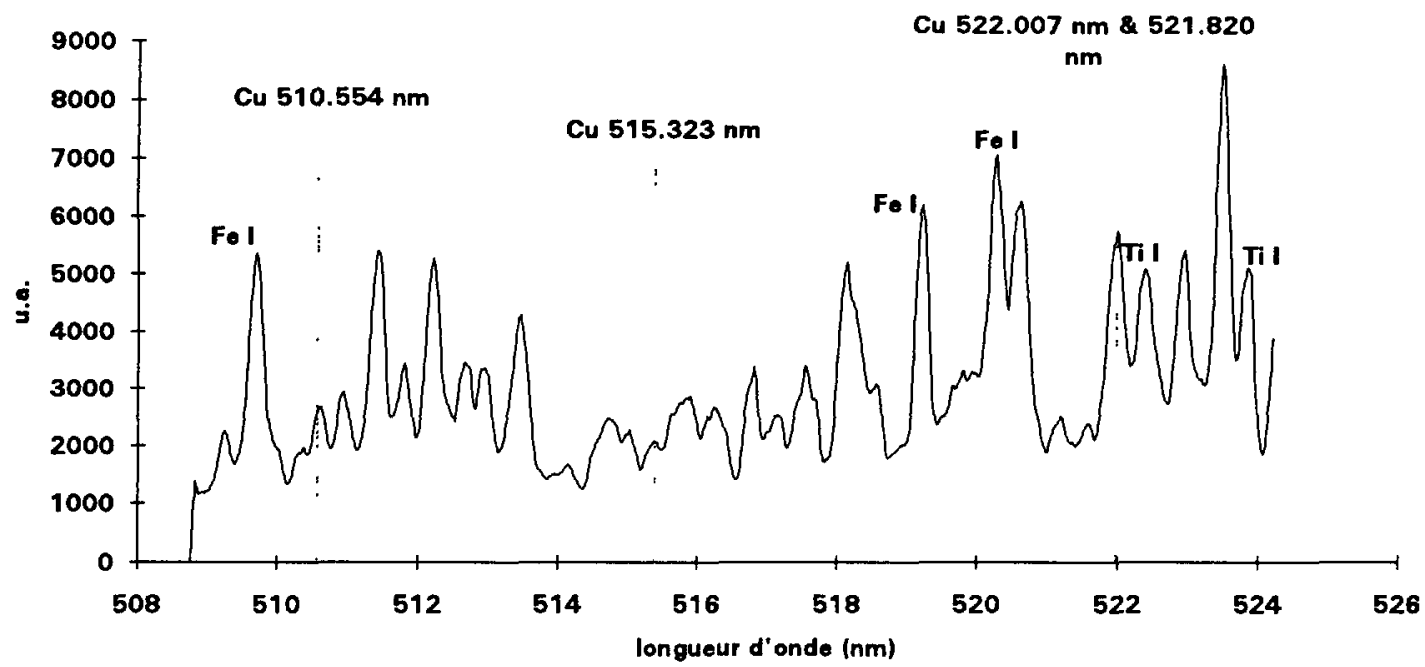

Fig. 15. - Spectre obtenu avec la poudre de titane sur la billette d'acier.

[Titanium powder spectrum.] 
$1 \mathrm{~mm} / \mathrm{s}$, ne permet d'observer le plasma créé dans le four que pendant quelques minutes, ce qui interdit une reconnaissance complète des espèces. Les premiers spectres ainsi obtenus se sont révélés inutilisables. En effet, le mélange des spectres (Fig. 15) émis par le fer de la billette et le titane en rendait l'interprétation complexe. La durée d'observation étant de quelques minutes, une identification systématique était impossible. En outre, la poussière dégagée par la poudre de titane induisait une forte chute de la transmission du rayonnement. Le cuivre a alors été choisi comme traceur au lieu du titane, notamment en raison des bons résultats qu'il a permis d'obtenir sur la torche à plasma.

La billette étant trop usée au centre, de nouveaux essais ont été réalisés en déposant quelques plaques de cuivre dans l'usure d'acier (Fig. 11). La vaporisation du titane ayant été très rapide lors de l'essai précédent, l'entregistrement des spectres émis par l'arc électrique a débuté dès la mise sous tension du four : la durée d'observation utile n'est plus que de quelques secondes. Sur les premiers spectres enregistrés (Fig. 16), les trois raies du cuivre intéressantes pour les mesures de températures sont bien distinctes du bruit de fond. Sur les enregistrements suivants (Fig. 17) au contraire, le flux émis par les atomes de fer se superpose à celui émis par les atomes de cuivre rendant l'analyse impossible. Au bout d'une trentaine de secondes environ, il ne subsiste que le flux émis par les atomes de fer, les plaques de cuivre ayant été complètement vaporisées. Dans le spectre du fer on n'a pas identifié de raies bien adaptées à la mesure de température dans la même fenêtre spectrale. Le choix du cuivre se révèle donc être le plus favorable aux mesures, mais avec la limitation d'un temps d'acquisition des spectres réduit.

La dynamique importante et les temps d'observation courts (de l'ordre de la dizaine de secondes) empêchent d'adapter le gain au signal. En raison du fort rayonnement, il a été nécessaire de placer un filtre neutre de densité 4. Les temps d'acquisition sont portés à $100 \mathrm{~ms}$. C'est le temps qui permet quelques observations visuelles à l'expérimentateur sur les phénomènes en cours. Sans le filtre, il aurait été possible, avec le même gain, de diminuer le temps d'acquisition à $10 \mu \mathrm{s}$. Un tel intervalle de temps aurait permis de visualiser la diffusion

\section{Raies du Cuivre}

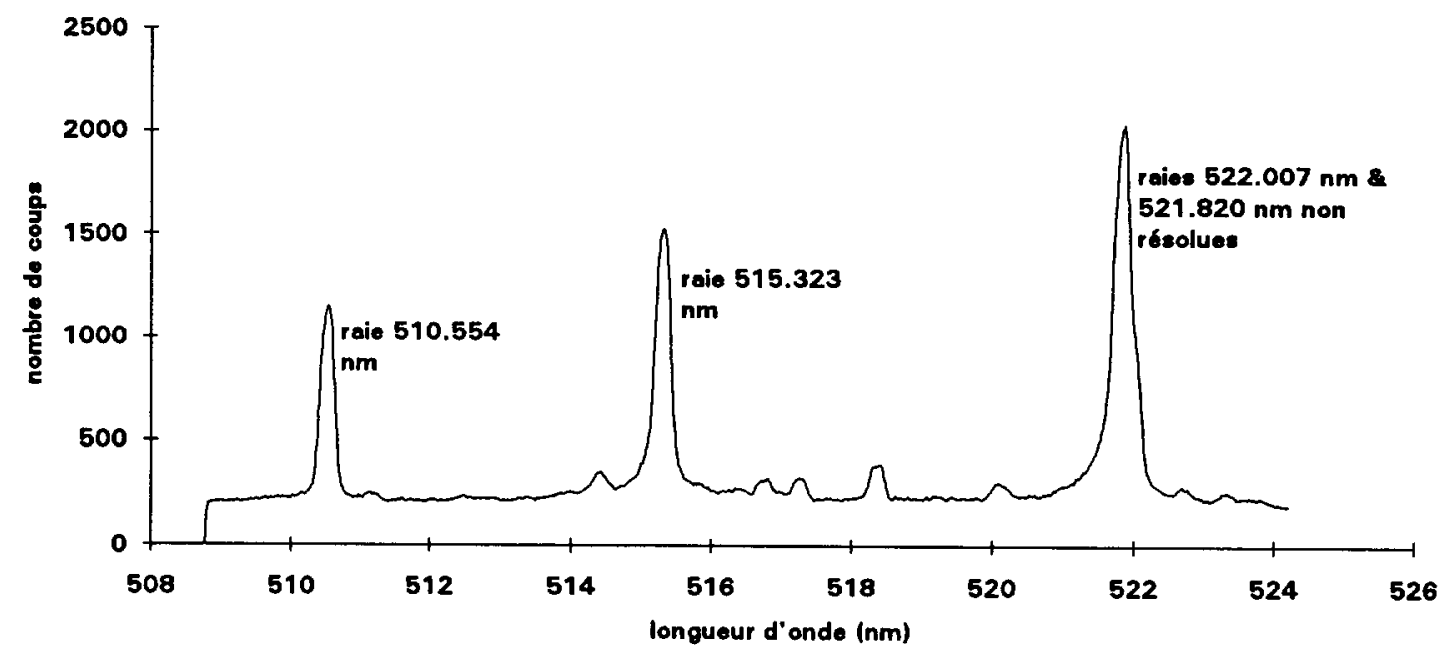

Fig. 16. - Spectre obtenu avec le cuivre sur la billette d'acier. Sur les premiers spectres, les raies du cuivre sont évidentes.

[Spectrum obtained by copper deposition on the steel blocs. The copper lines are obvious on the first spectra.] 


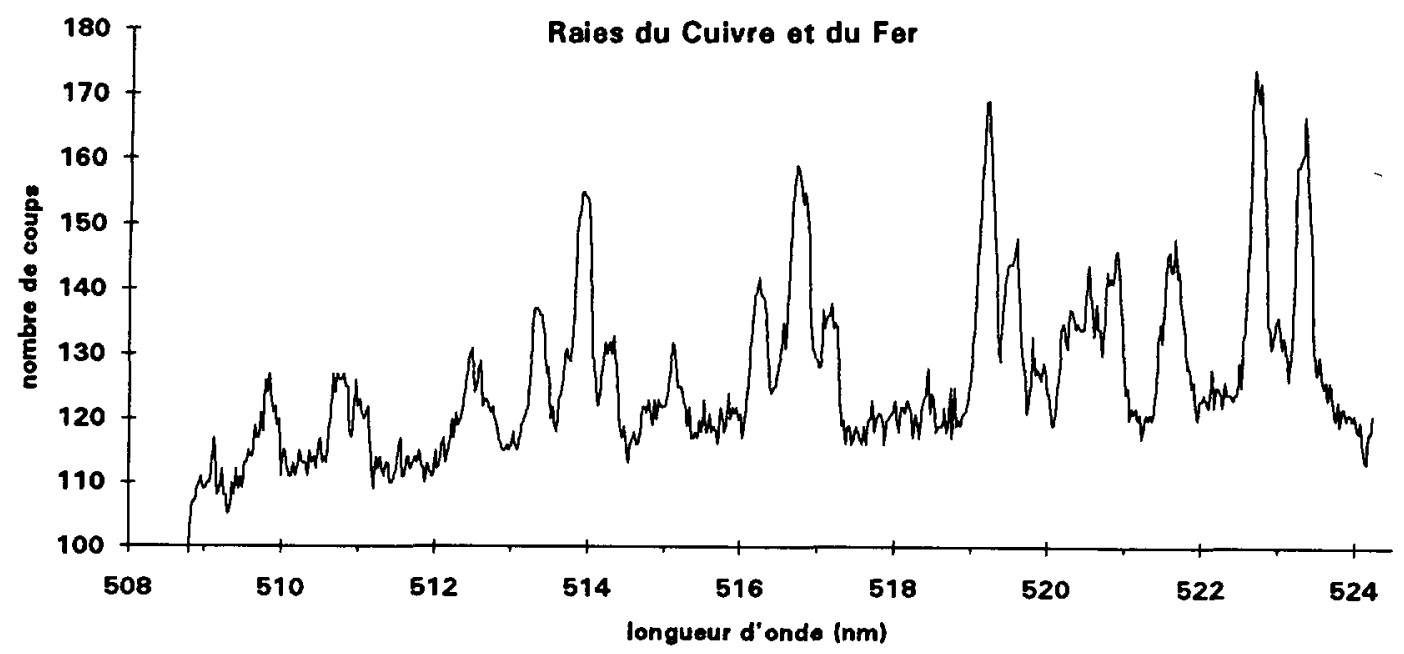

Fig. 17. - Sur les spectres suivants, les raies du cuivre se mélangent à celles du fer.

[On the next spectra, the copper lines overlap with iron lines.]

verticale du traceur dans le four. Les $100 \mathrm{~ms}$ choisies sont en effet de l'ordre de grandeur, voire supérieures au temps nécessaire pour la diffusion des atomes de cuivre du bas du four vers le haut : $20 \mathrm{~cm}$ parcourus en $100 \mathrm{~ms}$ donnent une vitesse de $2 \mathrm{~m} . \mathrm{s}^{-1}$, ce qui est tout à fait réaliste.

Un autre phénomène est intervenu lors de ces mesures. Le point d'accrochage inférieur de l'arc, où est déposé le cuivre, est très mobile. Or le détecteur intègre dans le temps les intensités. Les résultats sont alors proportionnels au temps de passage de l'arc dans la zone d'observation. Ceci induit de fortes variations d'intensité le long de l'axe vertical dans un rapport de 5 à 75 selon les mesures (Fig. 18). Le gain a alors été adapté aux intensités élevées et

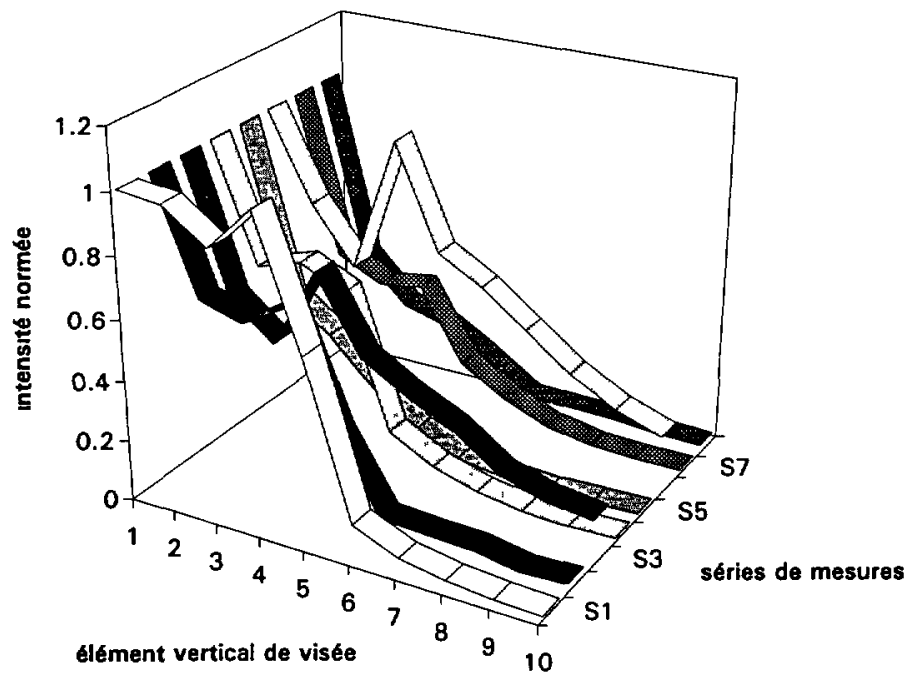

Fig. 18. - Dans le four à arc, les différentes intensités mesurées, normalisées, mettent en évidence l'instabilité de l'arc.

[In the electric furnace, the different normalized measured intensities feature the arc instability.] 


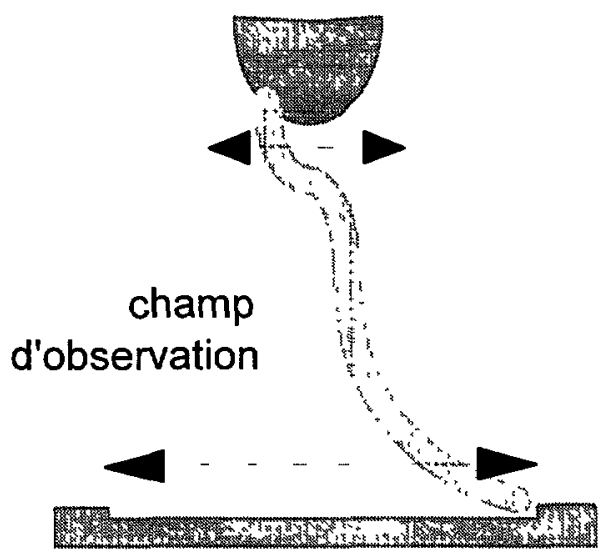

Fig. 19. - La représentation de l'instabilité de l'arc montre que celui-ci est plus souvent présent dans la partie supérieure d'observation que dans la partie inférieure.

[A representation of the arc instability shows that it is present more often in the higher part of the observation region than in the lower part.]

relativement constantes des zones supérieures, près de l'électrode, où l'arc est quasiment stable. Cependant, certaines séries de mesures présentant une saturation au niveau de l'électrode supérieure ont conduit à penser que la position quasi-stable de l'arc est en fait décentrée par rapport à la zone de visée, et que celui-ci effectue parfois de brèves incursions dans le centre du champ. La figure 18 interprétée en termes de position donne une position moyenne équivalente de l'arc (Fig. 19), sans que l'on puisse affirmer que celle-ci correspond à une position moyenne réelle de l'arc. Des temps de mesures de $10 \mu \mathrm{s}$ permettraient de visualiser les déplacements et les positions instantanées de l'arc dans le champ de visée.

L'arc a une largeur de $1 \mathrm{~cm}$ et est observé dans un champ large de $5 \mathrm{~cm}$, à grande distance. Comme il s'agit d'un milieu très localisé et présentant une forte dynamique, l'inversion d'Abel ne se justifie pas : la température la plus élevée masque en effet les températures les plus basses (Tab. II). En outre, si le milieu est fortement instable, il a peu de chance de présenter une

Tableau II. - Dans le champ d'observation, les températures les plus élevées masquent les plus faibles.

[In the observation region, the highest temperatures mask the lowest ones.]

\begin{tabular}{|c|c|c|c|c|}
\hline & \multicolumn{2}{|c|}{ Nombre de coups } & & \\
\hline Raie & à $8000 \mathrm{~K}$ & à $16000 \mathrm{~K}$ & Total & $\begin{array}{c}\text { Température } \\
\text { résultante }\end{array}$ \\
\hline 1 & $5 \mathrm{e} 12$ & $3,5 \mathrm{e} 13$ & $4,00 \mathrm{e} 13$ & \\
\cline { 1 - 4 } 2 & $5 \mathrm{e} 12$ & $2 \mathrm{e} 14$ & $2,05 \mathrm{e} 14$ & $\approx 16000 \mathrm{~K}$ \\
\hline 3 & $1 \mathrm{e} 13$ & $4 \mathrm{e} 14$ & $4,10 \mathrm{e} 14$ & \\
\hline
\end{tabular}


symétrie cylindrique. La température sera donc directement déduite des intensités mesurées. Une étude analogue d'une flamme de méthane dopée par un arc électrique avait montré les mêmes conclusions: au voisinage de l'arc, l'inversion d'Abel ne se justifie pas [2].

Le gain choisi porte à environ 2000 coups l'intensité moyenne mesurée pour la raie la plus intense (Fig. 16). Les calculs sur la dynamique exposés au paragraphe 3.1 donnent alors une température maximale détectable avant saturation de $20000 \mathrm{~K}$ environ, et une température minimale de $8000 \mathrm{~K}$, limite imposée par la deuxième raie. L'ensemble des mesures donne une température moyenne de l'arc comprise entre $12000 \mathrm{~K}$ et $16000 \mathrm{~K}$. Les variations de la température sur la hauteur de l'arc diffèrent selon les mesures : de quelques centaines de degré jusqu'à $3000 \mathrm{~K}$ environ. La gamme de températures détectables s'étendant suffisamment de part et d'autre de la valeur moyenne, il est certain que la température réelle est proche des valeurs trouvées, sans qu'il y ait à craindre de biais de mesures (saturation, sous-détection). Des températures proches de la limite inférieure de détection de $8000 \mathrm{~K}$ ont d'ailleurs été observées. La différence entre les $20000 \mathrm{~K}$ attendus et les $16000 \mathrm{~K}$ observés peut provenir de l'utilisation du cuivre comme métal au lieu du seul fer initialement envisagé dans les calculs. L'origine de l'écart en température peut être la différence de conductivité entre les deux métaux. Quoiqu'il en soit, il faudra tenir compte du $\mathrm{CO}_{2}$ pour modéliser les phénomènes en jeu.

\section{Conclusion.}

Les progrès dans la technologie des détecteurs et dans l'automatisation des procédures d'acquisition redonnent à la spectroscopie d'émission un nouvel attrait, auquel s'ajoute toujours sa simplicité de mise en œuvre. Pour les mesures de température, nous recommandons l'utilisation du cuivre comme traceur, qui permet des mesures aisées dans la gamme 2 500$25000 \mathrm{~K}$.

L'exemple d'utilisation sur la torche à plasma (milieu stationnaire) montre que les inconvénients majeurs de cette méthode de mesure (intégration spatiale, répartition du traceur) peuvent être levés, et que leur examen peut fournir des renseignements intéressants sur le comportement du milieu.

L'exemple d'utilisation sur un arc électrique montre que les inconvénients majeurs précédents disparaissent : les températures chaudes l'emportent sur les températures moyennes environnantes. Pour des observations de milieu instationnaire, l'utilisation d'une caméra 2D permet, là encore, de localiser les phénomènes.

Il faut donc considérer cette méthode comme un outil indispensable, dont la facilité de mise en cuvre en fait le complément idéal de méthodes de diagnostic plus sophistiquées.

\section{Bibliographie}

[1] Bacri J., Vacquié S., Méthodes de mesure applicables aux plasmas d'arc, RCP 369 : Arc Electrique, RGE, tome 88, n 6 (juin 1979).

[2] Cahen C., Sassi M., Temperatures in a turbulent diffusion flame with and without exposure to an electric arc, J.Q.S.R.T. 49 (1993) 281-301.

[3] Hong D., Diagnostic de plasmas denses, Thèses de l'Université d'Orléans (mars 1991).

[4] Griem H. R., Plasma spectroscopy (McGraw-Hill Book Co, 1964). 
[5] Yan W. Z., Wu C. K., On the diagnosis of arc plasma jets, Plasma jets in the development of new materials technology, Proc. of the International Workshop (VSP, 1990) pp. 125-131.

[6] Gellert B., Shade E., Dullni E., Measurement of particles and vapour density after high-current vacuum arc by laser techniques, XIIth Intern. Sym. on Dis. and Elec. Insul. in Vac., IEEE Proceedings (1986) 209-213.

[7] Radzig A. A., Smirnov B. M., Reference data on atoms, molecules, and ions (Springer-Verlag, 1985, origin. 1980) p. 209.

[8] Weast R. C. Ed.. CRC handbook of chemistry and physics (62e Ed. CRC Press, 1981-1982).

[9] Spiberg P., Diagnostic laser (Thermométrie DRASC, Vélocimétrie d'un générateur de plasma industriel : confrontation mesures-modèles, Thèse de l'Université de Paris 6 (1988).

[10] Cahen C., Spiberg P., Laser diagnosis inside a $2 \mathrm{MW}$ plasma generator, Plasma jets in the development of new materials technology, Proc. of the International Workshop (VSP, 1990) pp. 149-161.

[11] Bockasten K., Transformation of observed radiances into radial distribution of the emission of a plasma, J. Opt. Soc. Am. 51, n 9 (1961).

[12] Barr W. L., Method for computing the radial distribution of emitters in a cylindrical source, J. Opt. Soc, Am. 52, n 8 (1962).

[13] Maldonado C. D., Carn A. P., Olsen H. N., New method of obtaining emission coefficients from emitted spectral intensities: Part I Circularly symmetric light sources, J. Opt. Soc. Am. 55 (1965).

[14] Cremers C. J., Birkebak R. C., Application of the Abel integral equation to spectrographic data, Appl. Opt. 5, n 6 (1966).

[15] Glaser J., Chapelle J., Boettner J. C., Abel inversion applied to plasma spectroscopy : a new interactive method, Appl. Opt. 17, n 23 (1978).

[16] Deutsch M., Beniaminy I., Derivative-free inversion of Abel's integral equation, Appl. Phys. Lett. 41 (1) (1982).

[17] Deutsch M., Beniaminy I., Inversion of Abel's integral equation for experimental data, J. Appl. Phys. 54 (1) (1983).

[18] Deutsch M., Abel inversion with a simple analytic representation for experimental data, Appl. Phys. Lett. 42 (3) (1983).

[19] Hansen E. W., Law P. L., Recursive methods for computing the Abel transform and its inverse, $J$. Opt. Soc, Am. 2, n 4 (1985).

[20] Tatum J. B., Jaworski W. A., A solution of Abel's equation, J.Q.S.R.T. 38 (1987) 319.

[21] Vicharelli P. A., Lapatovich W. P., Iterative method for computing the inverse Abel transform, Appl. Phys. Lett. 50 (10) (1987).

[22] Kalal M., Nugent K. A., Abel inversion using fast Fourier transforms, Appl. Opt., 27, n 10 (1988).

[23] Deutsch M., Notea A., Pal D., Reconstruction -of discontinuous density profiles of cylindrically symmetric objects from single X-ray projections, Appl. Opt. 27, n 19 (1988).

[24] Deutsch M., Notea A., Pal D., Abel reconstruction of piecewise constant radial density profiles from X-ray radiographs, Appl. Opt. 28, n 15 (1989).

[25] Press W. H., Flannery B. P., Teukolsky S. A., Vetterling W. T., Numerical recipes : the art of scientific computing (Cambridge University Press, 1986).

[26] Chyou Y. P., Pfender E., Modelling of plasma jets with superimposed vortex flow, Plasma Chem. Plasma Process. 9 (1989) 291.

[27] Brossa M., Pfender E., Probe measurements in thermal plasma jets, Plasma Chem. Plasma Process 8 (1988) 75.

[28] Capetti A., Pfender E., Probe measurements in argon plasma jets operated in ambient argon, Plasma Chem. Plasma Process. 9 (1989) 329.

[29] Spores R., Pfender E., Flow structure of a turbulent thermal plasma jet, Surf. Coating Technol. 37 (1989) 251.

[30] Chen W. L. T., Pfender E., Two-dimensional temperature measurements in thermal plasma jets using an OMA system, 9th International Symposium on Plasma Chemistry, Vol. I (Pognochiuso, September 1989). 
[31] Chen W. L. T., Pfender E., Enthalpy probe and spectroscopic measurements in thermal plasma jets, ERC for Plasma-Aided Manufacturing, Department of Mechanical Engineering (University of Minnesota, April 1990).

[32] Chen W. L. T., Heberlein J., Pfender E., Experimental measurements of plasma properties for Miller SG-100 torch with Mach I setting, ERC for Plasma-Aided Manufacturing, Department of Mechanical Engineering (University of Minnesota, November 1990).

[33] Pfender E., Chen W. L. T., Chiu C. P., Heberlein J., Studies of a turbulent argon-helium plasma jet (NTSC, Pittsburgh PA, May 1991).

[34] Pfender E., Finck J., Spores R., Entrainment of cold gas into thermal plasma jets, Plasma Chem. and Plasma Process. 11, n 4 (1991).

[35] Veillette P., Simard D., Electric arc characterization using high speed cinematography and spectroscopy, 42nd Electric Furnace Conference of Iron and Steel Society (Toronto 1984). 